\title{
Electrogenerated chemiluminescence of violanthrone in dimethyl sulfoxide
}

\author{
K. Hansongnern, P. Chooto, and P. Amornpitoksuk \\ Department of Chemistry, Faculty of Science, Prince of Songkla University, \\ Hat-Yai, Songkla 90112, Thailand
}

\begin{abstract}
Violanthrone is an anthraquinone vat dye, which is resistant to photochemical damage. Most previous work has concentrated on its chemiluminescence properties. In this report, electrogenerated chemiluminescence (ECL) of violanthrone has been investigated in dimethyl sulfoxide with $0.1 \mathrm{M}$ of tetraethylammonium bromide as electrolyte. Violanthrone luminesces in the potential range between $0.0 \mathrm{~V}$ and $-4.0 \mathrm{~V}$. At the sweep rate $1000 \mathrm{mV} / \mathrm{s}$, the intensity of the violanthrone ECL is about $2 \%$ of that of tris(2,2'-bipyridine) ruthenium(II) ion under the same conditions. It was found that bromine was also produced during the experiment, leading to the conclusion that the luminescence arises from the reaction between radical anion of violanthrone and molecular bromine. The mechanism is discussed in detail.
\end{abstract}

\section{INTRODUCTION}

Chemiluminescence of some reactions have been studied and it was found that the excited states are generated by electron-transfer processes and the light was observed at the electrode surfaces. This is called electrogenerated chemiluminescence [1]. One route to ECL is via an ion-annihilation reaction mechanism. For example, the reactions between radical anions and cations of 9,10-diphenylanthracene have been studied in acetonitrile, propylene carbonate or the mixtures of solvents [2]. Some involved the reactions between strong reductants $\left(A^{-\cdot}\right)$ and strong oxidants $\left(D^{+\cdot}\right)$ which are generated electrochemically such as the ECL of the $\left[\mathrm{Ru}(\mathrm{bpy})_{3}\right]^{2+}-\mathrm{S}_{2} \mathrm{O}_{8}^{2-}$ system in acetonitrile-water solutions [3]. The excited state $\left[\mathrm{Ru}(\mathrm{bpy})_{3}\right]^{2+}$ is generated via oxidation of $\left[\mathrm{Ru}(\mathrm{bpy})_{3}\right]^{+}$by $\mathrm{SO}_{4}^{-}$. ECL of violanthrone has been investigated in dimethylformamide at sweep rate $80 \mathrm{~V} / \mathrm{s}$ in $0.1 \mathrm{M}$ with tetrabutylammonium bromide (TEAB) as electrolyte, in the potential range $-4.0 \mathrm{~V}$ to $-4.0 \mathrm{~V}$ [4]. The mechanism was proposed to be the result of annihilation of the radical anions and radical cations of violanthrone produced electrochemically.

In this work, we investigated the ECL of violanthrone in dimethyl sulfoxide, but in the potential range $0.0 \mathrm{~V}$ to $-4.0 \mathrm{~V}$ with $\mathrm{TEAB}$ as electrolyte. The luminescence was found to be produced via a route different from that previously reported.

\section{MATERIALS AND METHODS}

Materials. The ECL of violanthrone was studied using a deoxygenated solution in dimethyl sulfoxide (Carlo Erba reagent) with a supporting electrolyte of tetraethyl-ammonium bromide (TEAB, Aldrich) at a concentration of $0.1 \mathrm{~mol} / \mathrm{L}$. Violanthrone was obtained from BASF and used as received.

Electrochemical measurements. Cyclic voltammetric investigations of violanthrone at a platinum electrode were done in dimethyl sulfoxide containing 0.1 M TEAB. The electrochemical measurements were carried out us- ing MacLab /4 e, MacLab potentiostat from ADInstrument Pty Ltd., connected to Macintosh PowerPC Performa 7300/120 computer, with the EChem Program version 1.3.2 to control the applied potentials for electrochemical reactions. Platinum disk is a working electrode. Pt wire is an auxiliary electrode and the reference is a $\mathrm{Ag} / \mathrm{AgCl}$ electrode which was calibrated to the saturated calomel electrode (SCE). Then all potentials are reported to SCE and the sweep rate is $200 \mathrm{mV} / \mathrm{s}$

Electrogenerated chemiluminescence measurements. For all ECL experiments, the sweep rate was set at $1000 \mathrm{mV} / \mathrm{s}$ in the potential range $0.0 \mathrm{~V}$ to $-4.0 \mathrm{~V}$. The Scope Program was used to generate the waveform within a given period of time. The area under each waveform was determined by the Peak Program supplied by the ADInstrument Pty Ltd. The $3 \mathrm{~mL}$ of solution was transferred to the quartz cell, with a diameter of $1 \mathrm{~cm}$. All three electrodes were immersed in the cell. The working electrode is Pt foil, $0.9 \times 0.5 \mathrm{~cm}$, total area, $0.45 \mathrm{~cm}^{2}$. The auxiliary is also Pt foil, $1.4 \times 1.5 \mathrm{~cm}$ total area $2.1 \mathrm{~cm}^{2}$. Pt wire was used as a quasi-reference electrode. All solutions were degassed with argon and measurements were carried out at room temperature, $25^{\circ} \mathrm{C}$. The relative ECL intensity measurements were performed using Jasco FP-777 spectrofluorometer. Integration of the ECL intensity over finite periods of time was accomplished using the program supplied by Jasco Company. The ECL intensity from violanthrone was compared to that of $\left[\mathrm{Ru}(\mathrm{bpy})_{3}\right](\mathrm{PF})_{6}$ under the same conditions.

\section{RESULTS AND DISCUSSION}

Violanthrone is a vat dye, used in fabrics and is known for its resistant to photochemical damage. It is an aromatic hydrocarbon with a lot of conjugated systems of double bonds as shown in Figure 1. Violanthrone gives emission in many nonaqueous solvents such as dimethyl sulfoxide and the maximum wavelength of luminescence is $626 \mathrm{~nm}$. The electrochemistry of violanthrone was studied in dimethyl sulfoxide 


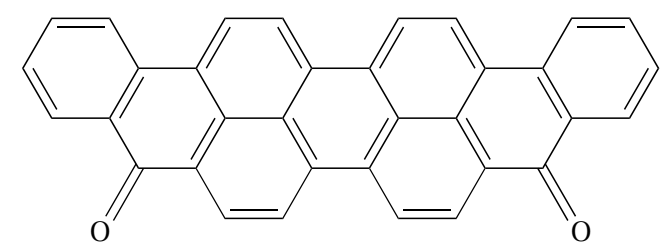

Figure 1. The structure of violanthrone.

with $0.1 \mathrm{M}$ TEAB as electrolyte. The cyclic voltamogram is shown in Figure 2. One couple in the reduction wave occurred with the $\mathrm{E}_{\mathrm{pc}}=-0.45 \mathrm{~V}$ and the $\mathrm{E}_{\mathrm{pa}}=-0.39 \mathrm{~V}$ at a Pt electrode. The potentials are $v s$. the saturated calomel electrode.

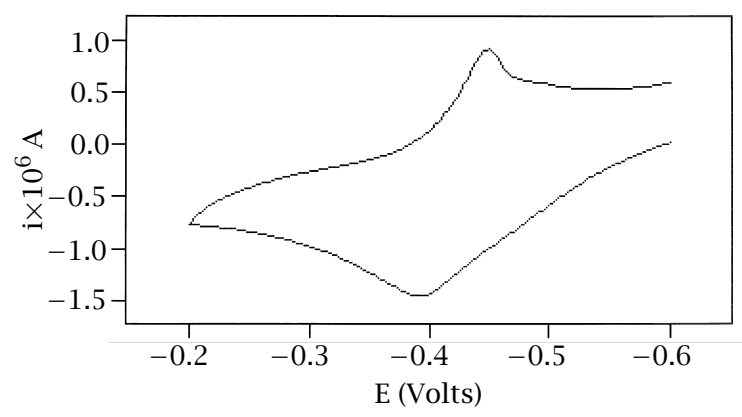

Figure 2. Cyclic voltammetry of violanthrone in dimethyl sulfoxide with 0.1 M TEAB as electrolyte (Pt-disk working electrode). All potential are vs. the saturated calomel electrode (SCE) at a scan rate of $200 \mathrm{mV} / \mathrm{s}$.

For ECL experiments, the electrodes used are different from normal cyclic voltammetry mentioned above. Pt foil, the auxiliary electrode, has greater surface area than that of the working electrode (Pt foil) and Pt wire was used as quasi-reference electrode. This setup is a requirement for the ECL experiments in order to generate the large amount of the radical anions produced electrochemically. In the previous work we studied the ECL experiments of violanthrone in dimethylformamide in the potential range $+4.0 \mathrm{~V}$ to $-4.0 \mathrm{~V}$ [4]. However, in this work we confine the investigation of ECL study to the electrical measurement in the potential range $0.0 \mathrm{~V}$ to $-4.0 \mathrm{~V}$ in order to obtain more information about the mechanism. The luminescence intensity from ECL was detected at $626 \mathrm{~nm}$.

Emission was only detected from the DMSO solution of violanthrone when the potential applied to the working electrode was cycled from $0.0 \mathrm{~V}$ to a sufficiently negative value. The scan rate was kept constant at $1000 \mathrm{mV} / \mathrm{s}$ because the detector of spectrofluorometer gives the best signal at that speed. In addition, when cycling the potentials between $0.0 \mathrm{~V}$ to $-2.5 \mathrm{~V}$ and beyond, molecular bromine was also seen as yellow liquid at the bottom of the quartz cell.

Figure 3 illustrates the behavior of violanthrone during the ECL experiments. During cycling the voltage ranges from $0.0 \mathrm{~V}$ to $-3.0 \mathrm{~V}$. Between $0.0 \mathrm{~V}$ and $-1.7 \mathrm{~V}$,

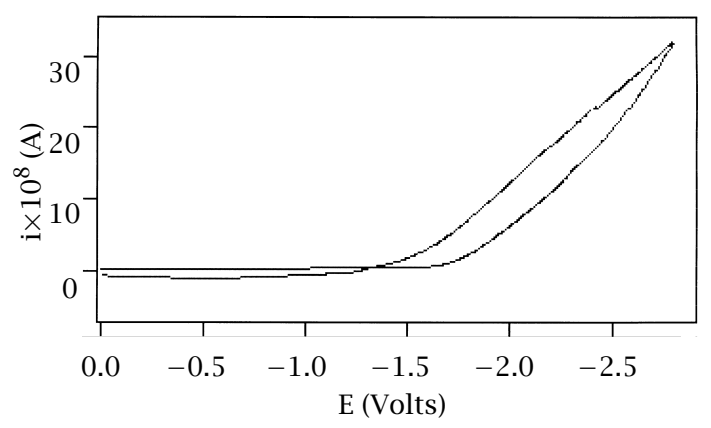

Figure 3. Cyclic voltammetry of violanthrone during ECL experiment (Pt-foil working electrode). The potential were cycled between $0.0 \mathrm{~V}$ and $-3.0 \mathrm{~V}$, relative to the $\mathrm{Pt}$ quasi-reference electrode, at a scan rate $1000 \mathrm{mV} / \mathrm{s}$. The supporting electrolyte is $0.1 \mathrm{M} \mathrm{TEAB}$.

a residual current was low. When the applied potential was greater than $-1.7 \mathrm{~V}$, the cathodic current increased markedly. Figure 4 shows that the potential limits effects on the intensity of the ECL produced by cycling the working electrode. The light intensity is still increasing with the increasing potential limits. The correlation between the potential limits and the emission gave linear relationship. This indicates that intensity of ECL was subject to the amount of ions produced; more ions generate greater current and more light intensity. The ECL of violanthrone should be due to the combination of ions to each other in the given potential range.

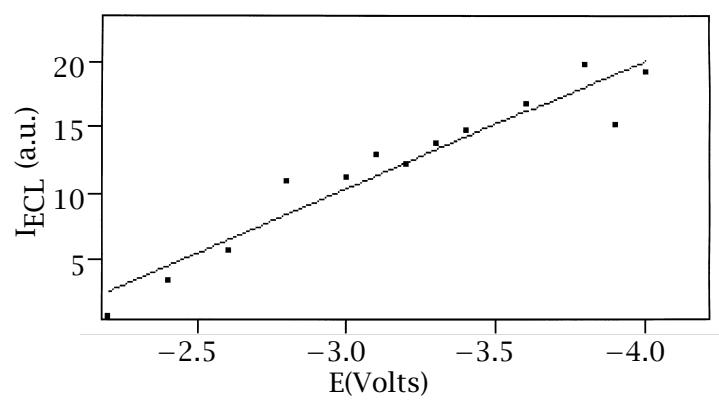

Figure 4. The effect of the potential limits on the intensity of the electrogenerated chemiluminescence, $I_{\mathrm{ECL}}$, produced by cycling the working electrode in the potential range $0.0 \mathrm{~V}$ to $-4.0 \mathrm{~V}$. All potentials are relative to the $\mathrm{Pt}$ quasi-reference electrode at a scan rate $1000 \mathrm{mV} / \mathrm{s}$ in $0.1 \mathrm{M} \mathrm{TEAB}$.

As seen from Figure 3, only a small residual current was observed until the potential limit reached $-1.8 \mathrm{~V}$, beyond this potential the current increased significantly. The reason for this behavior is that prior to this value, only $\mathrm{Br}^{-}$was present, while beyond that both $\mathrm{Br}_{2}$ and $\mathrm{Br}^{-}$were present. The reaction at the cathode was the reduction of violanthrone. This species was the precursor of radical anion of the emitter. Meanwhile, bromide ions can undergo oxidation at the auxiliary electrode. A possible sequence for the new mechanism can be written as follows:

Violanthrone $+\mathrm{e}^{-} \longrightarrow$ Violanthrone $\mathrm{V}^{-}$ 


$$
2 \mathrm{Br}^{-} \longrightarrow \mathrm{Br}_{2}+2 \mathrm{e}^{-} .
$$

At working elecrode, the reduction of violanthrone occurred, whereas the bromide ions were oxidized as bromine at the auxiliary electrode. Then the bromine molecules can act as an oxidizing agent which oxidized the radical anions of violanthrone as follows:

$$
\begin{gathered}
\text { 2Violanthrone }^{-\cdot}+\mathrm{Br}_{2} \longrightarrow \text { 2Violanthrone }{ }^{*}+2 \mathrm{Br}^{-} \\
\text {Violanthrone }^{*} \longrightarrow \text { Violanthrone }+h v .
\end{gathered}
$$

In addition, since the concentration of supporting electrolyte, TEAB, is $0.1 \mathrm{M}$, therefore, the bromine concentration is quite high compared to the concentration of violanthrone $\left(10^{-5} \mathrm{M}\right)$. In the presence of the species $\mathrm{Br}_{2}$ and $\mathrm{Br}^{-}$the reactions above can carry substantial current between electrodes as seen in Figure 3.

\section{CONCLUSIONS}

Violanthrone can produce the electrogenerated chemiluminescene in dimethyl sulfoxide by reductive oxidation. The radical anion of violanthrone was generated and reacted with the oxidizing agent, bromine molecule produced electrochemically. The studies are currently extended to other fluorescent compounds.

\section{ACKNOWLEDGEMENTS}

The authors gratefully acknowledge Thailand Research Fund (Grant No. BRG 4080001) for supporting this research.

\section{REFERENCES}

[1] L. R. Faulker and A. J. Bard, Electroanalytical Chemistry (A. J. Bard, ed.), vol. 10, Marcel Dekker, New York, 1977, p. 1.

[2] K. M. Maness, J. E. Bartelt, and R. M. Wightman, J. Phys. Chem. 98 (1994), 3993.

[3] H. S. White and A. J. Bard, J. Am. Chem. Soc. 104 (1982), 6891.

[4] T. I. Quickenden and K. Hansongnern, J. Biolumin. Chemilumin 10 (1995), 103. 


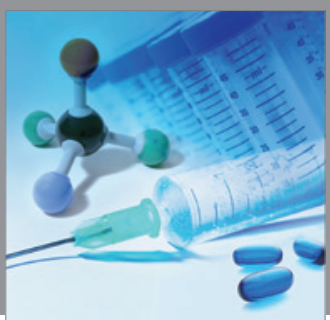

International Journal of

Medicinal Chemistry

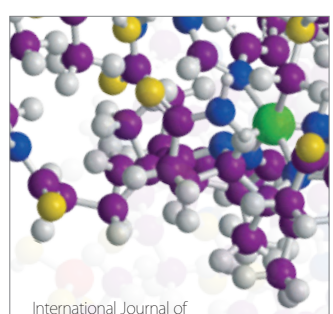

Carbohydrate Chemistry

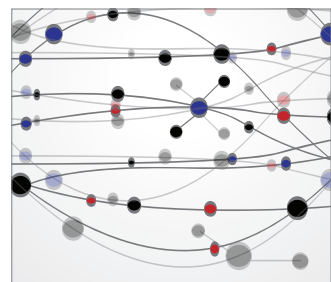

The Scientific World Journal
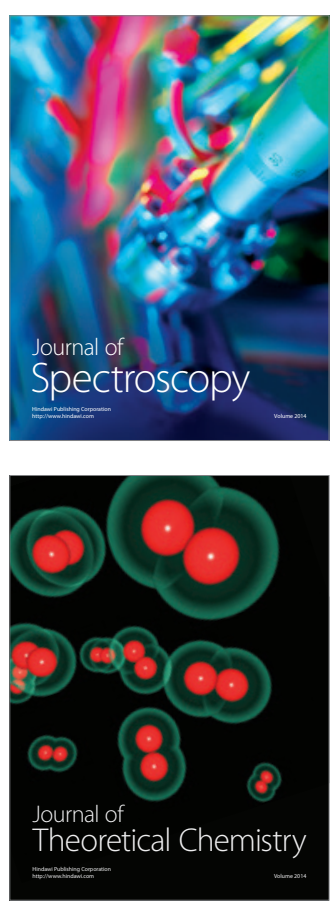
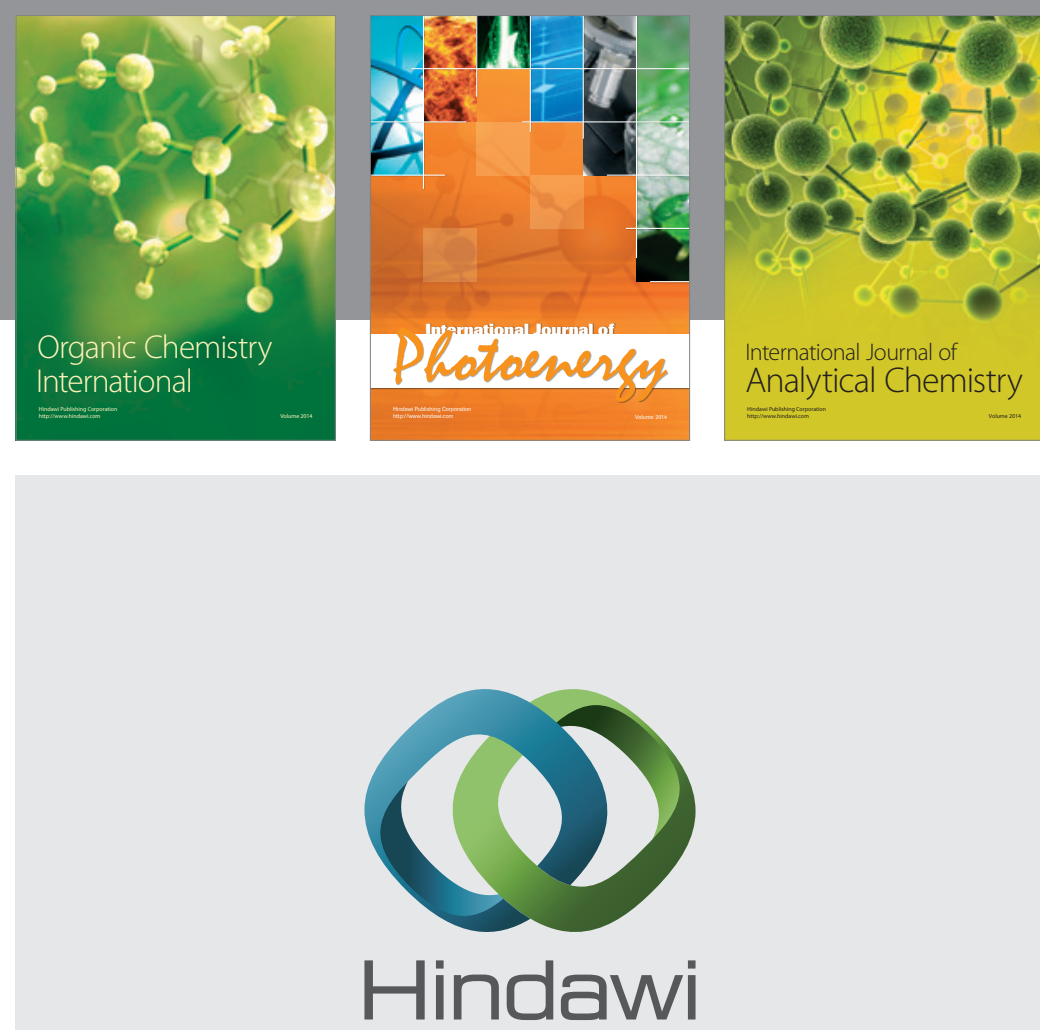

Submit your manuscripts at

http://www.hindawi.com
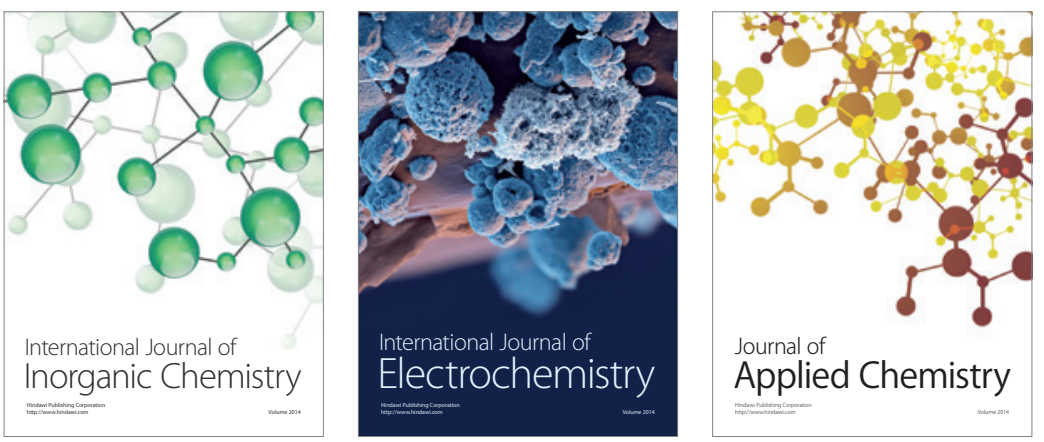

Journal of

Applied Chemistry
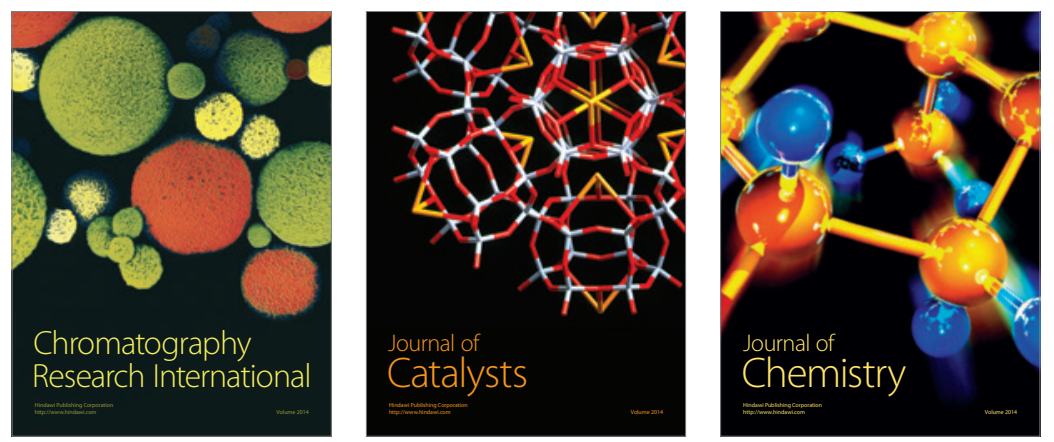
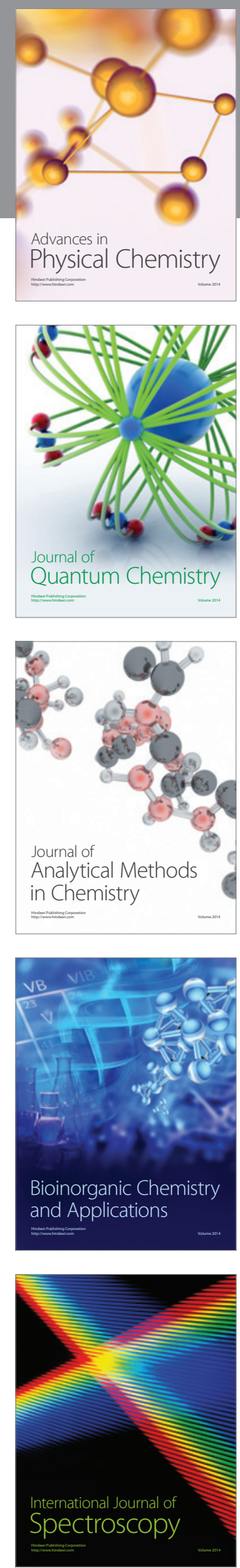